One-Pot Structure-Direction of Large-Pore Co-continuous Carbon Monoliths from Ultralarge Linear Diblock Copolymers

William R. T. Tait, ${ }^{1,2 \|}$ R. Paxton Thedford, ${ }^{1,2 \|}$ Dana V. Chapman, ${ }^{2}$ Fei $\mathrm{Yu}^{2,3}{ }^{2,3}$ Judson W. Freidl, ${ }^{1}$ Ekaterina S. Sablina, ${ }^{2}$ Gavin M. Batsimm, ${ }^{1}$ Ulrich B. Wiesner ${ }^{2 *}$

1. Robert Frederick Smith School of Chemical and Biomolecular Engineering, Ithaca, New York 14853, United States

2. Department of Materials Science \& Engineering, Cornell University, Ithaca, New York 14853, United States

3. Department of Chemistry and Chemical Biology, Cornell University, New York 14853, United States

$\|$ W.R.T.T. and R.P.T. contributed equally to this work.

* Corresponding author 
Table of Contents:

1. Static light scattering analysis

2. Proton nuclear magnetic resonance (NMR)

3. Estimates of degree of polymerization for each block of ULMM-SA1 and ULMM-SA2

4. Raman spectroscopy fitting and analysis

5. X-ray photoelectron spectroscopy (XPS) data

6. X-ray diffraction $(X R D)$ data 


\section{Static light scattering analysis}

It was found that the use of static light scattering (SLS) to create Zimm plots was necessary to determine the molar mass of the two PS- $b$-PDMAEMA polymers used in this study, as their respective ultralarge size and the interactions of the amine-containing block with the equipped column made traditional size exclusion chromatography (SEC) unreliable. The accuracy of SLS-derived, ultralarge molar mass values for ULMM-SA1 and ULMM-SA2 reported in the main text, and the validity of the method for interrogating isolated polymer coils in solution (as opposed to, e.g. micellar structures) is supported by various observations. First, during the course of polymerization the viscosity of the living polymer solutions increased drastically and substantially beyond what we typically observe for polymers with molar mass, e.g. around 100-200 kDa synthesized in our labs. This is consistent with behavior expected from the power law dependence of solution viscosity on polymer molar mass as described in the MarkHouwink equation and with molar masses of many 100 s of kDa we determined. Second, block copolymer synthesis efforts that 'failed' in adding a second block typically resulted in polystyrene homopolymers with molar masses $>500 \mathrm{kDa}$ as determined by SEC. Third, self-assembled structures obtained from coassembly of ULMM-SA1 and ULMM-SA2 with resols as shown in this work, as well as with other inorganic additives not described here, resulted in structural features of $100 \mathrm{~nm}$ or greater. This is clearly documented in the SEM images shown in the main text. Furthermore, independent SAXS measurements on these composite materials (data not shown) resulted in primary scattering peaks with positions well below the q-range typically accessible by conventional SAXS instruments. The non-linear dependance of domain size on polymer molar mass coupled with the high degree of shrinkage seen upon pyrolysis of $B C P$ composites implies such features could only be the result of BCP templating agents of $500+\mathrm{kDa}$ molar mass. The molar mass values determined by SLS as described in the main text are therefore consistent with our expectations for the mass of single polymer chains of ULMM-SA1 and ULMM-SA2, as opposed to that of micelle complexes, which by the same logic would have a substantially larger molar mass. Finally, while micellization occurred in final materials after full evaporation from pure THF solutions (e.g. see Figure $3 \mathrm{~B}$ in the main text), this only was observed for solutions containing much more highly segregating resols additives, and at starting concentrations (4 wt\%) more than an order of magnitude higher than the highest concentration used in our SLS measurements ( 0.12 wt\%). All of this strongly suggests that our values for molar mass of ULMM-SA1 and ULMM-SA2 on the order of 1,000 kDa as determined via Zimm plots from SLS experiments represent characteristics of single chains. 

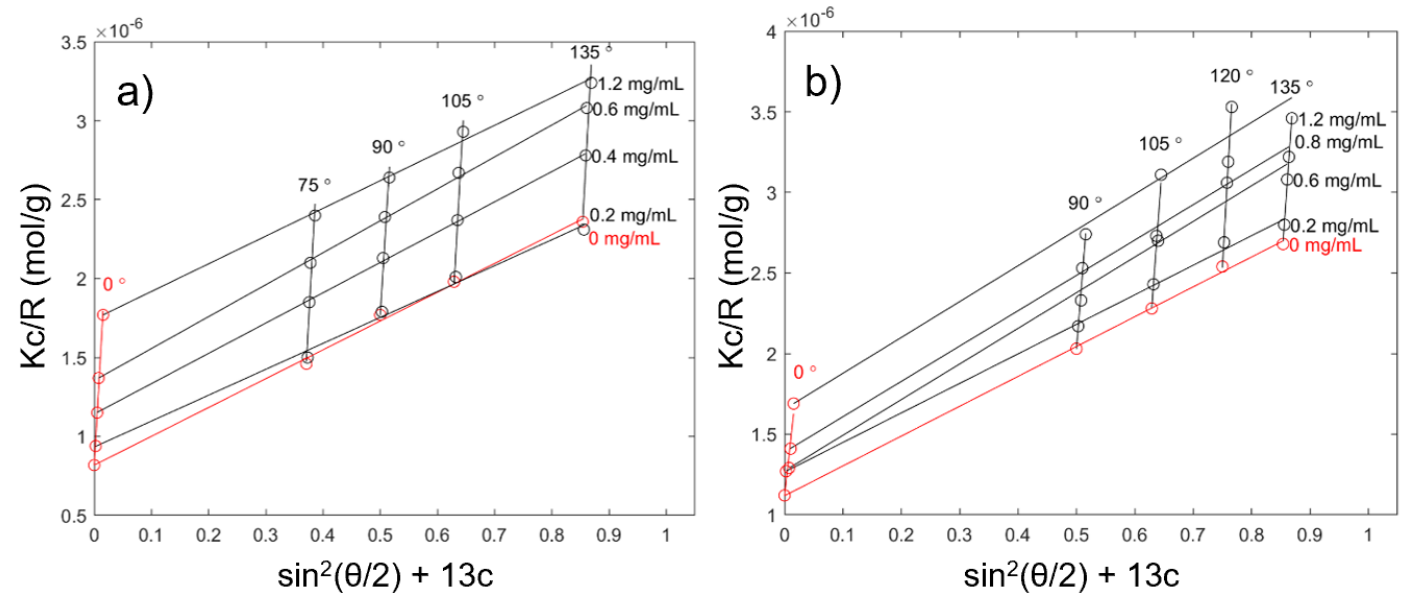

Figure S1: Independent set of results from Zimm plots of static light scattering results for a) ULMM-SA1 and b) ULMM-SA2.

\section{Proton nuclear magnetic resonance (NMR)}

a)

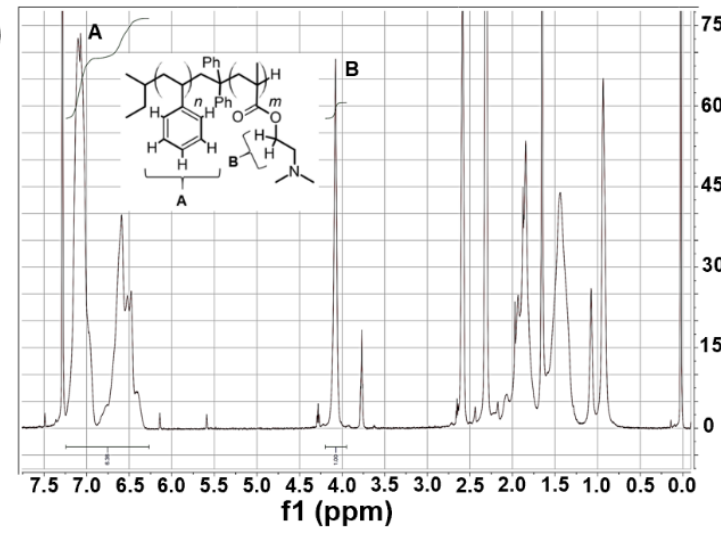

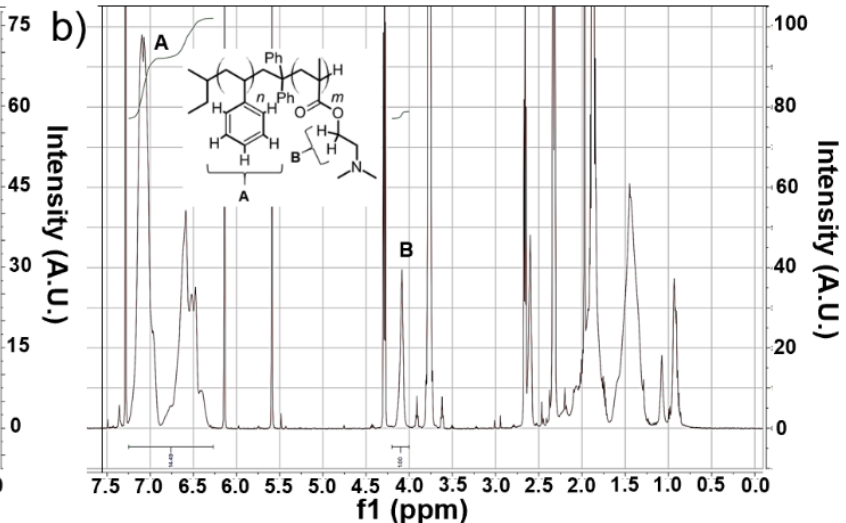

f1 (ppm)

Figure S2: ${ }^{1} \mathrm{H}$ NMR results for a) ULMM-SA1 and b) ULMM-SA2 to determine block fractions, where A and $B$ are the integrations associated with PS and PDMAEMA peaks, respectively.

\section{Estimates of degree of polymerization for each block of ULMM-SA1 and ULMM-SA2}

Taking the information from Table 1 in the main text, an estimate of the "weight average" degree of polymerization was calculated for each ULMM-SA; from that analysis, ULMM-SA1 and ULMM-SA2 could also be presented as $\mathrm{PS}_{7570}-b$-PDMAEMA 3020 and $\mathrm{PS}_{6870}-b$-PDMAEMA 1270 , respectively. Please note that these are overestimations of the commonly derived number average molar mass based degrees of polymerization. 


\section{Raman spectroscopy fitting and analysis}

$L_{\alpha}$, the graphitic cluster size, was derived via Equation $S 1$, which employed the values of $I_{D}$ and $I_{G}$ from integrating the curves used to fit the D-band and G-band in the experimental Raman spectra, respectively (Figure S3). A Lorentz function was used to fit the D-band, while a Breit-Wigner-Fano function was used to fit the G-band.

Equation S1: Empirical formula to determine the in-plane thickness of the graphitic sheets, $\mathrm{L}_{a}$, in angstrom:

$$
L_{a}=43.9\left(\frac{I_{D}}{I_{G}}\right)^{-1}
$$

a)
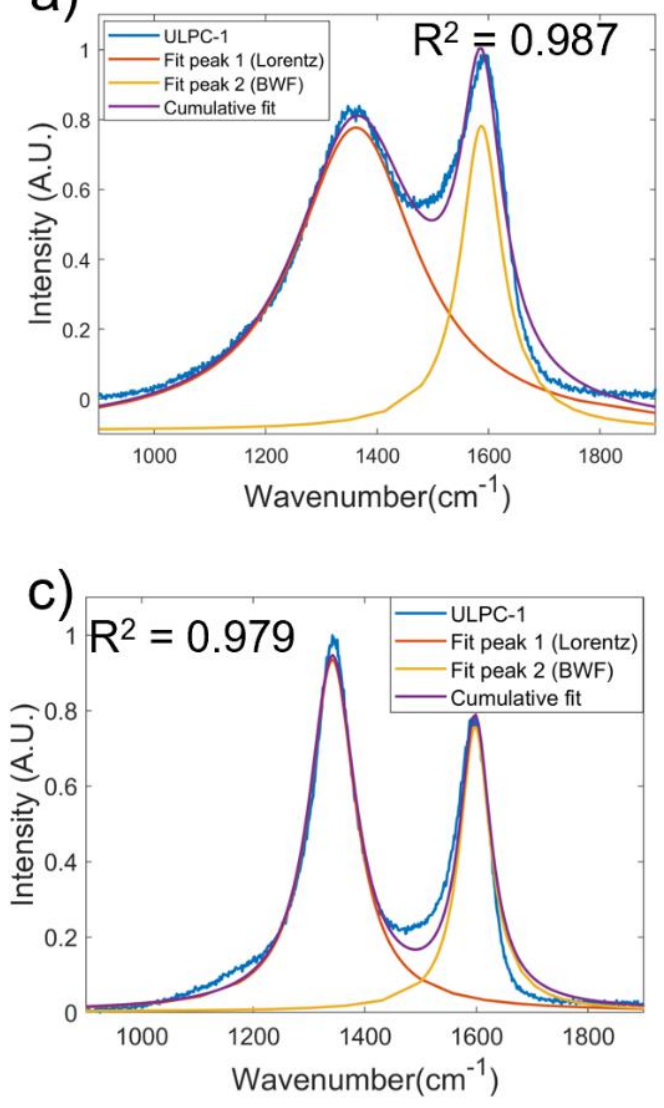
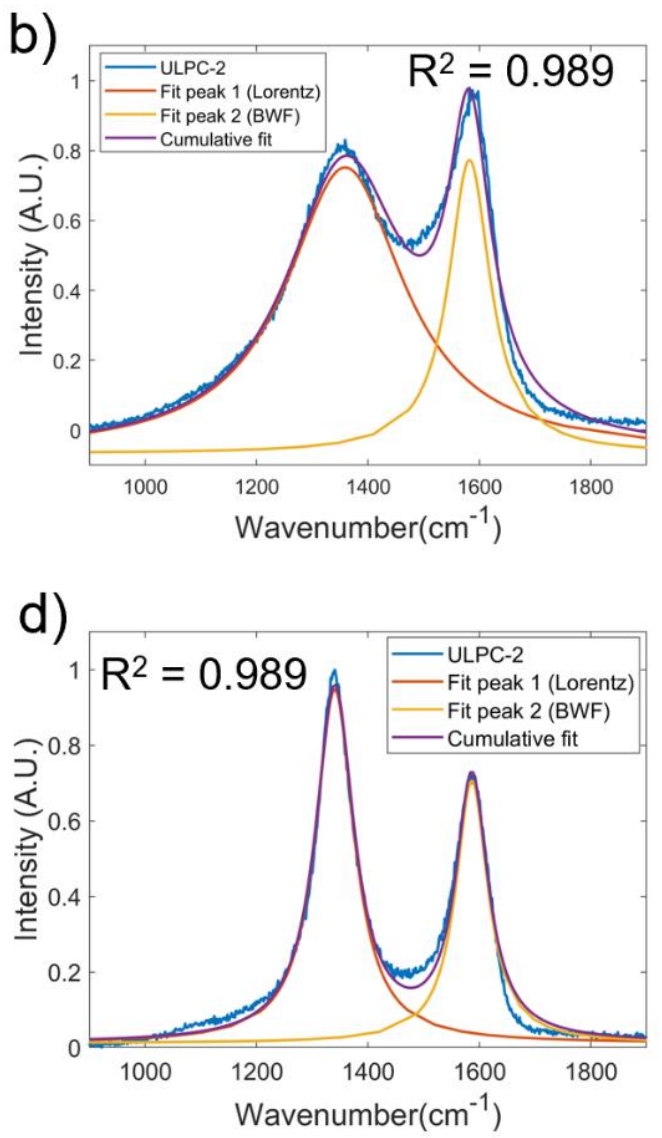

Figure S3: Raman spectra of a) ULPC- 1 and b) ULPC- 2 after pyrolysis at $900{ }^{\circ} \mathrm{C}$, as well as c) ULPC-1 and d) ULPC-2 after pyrolysis at $1600{ }^{\circ} \mathrm{C}$, together with fits using Lorentz functions of the D-bands and BreitWigner-Fano fitting of the $G$-bands. $R^{2}$ values for the cumulative fits are provided for each spectrum. 


\section{X-ray photoelectron spectroscopy (XPS) data}
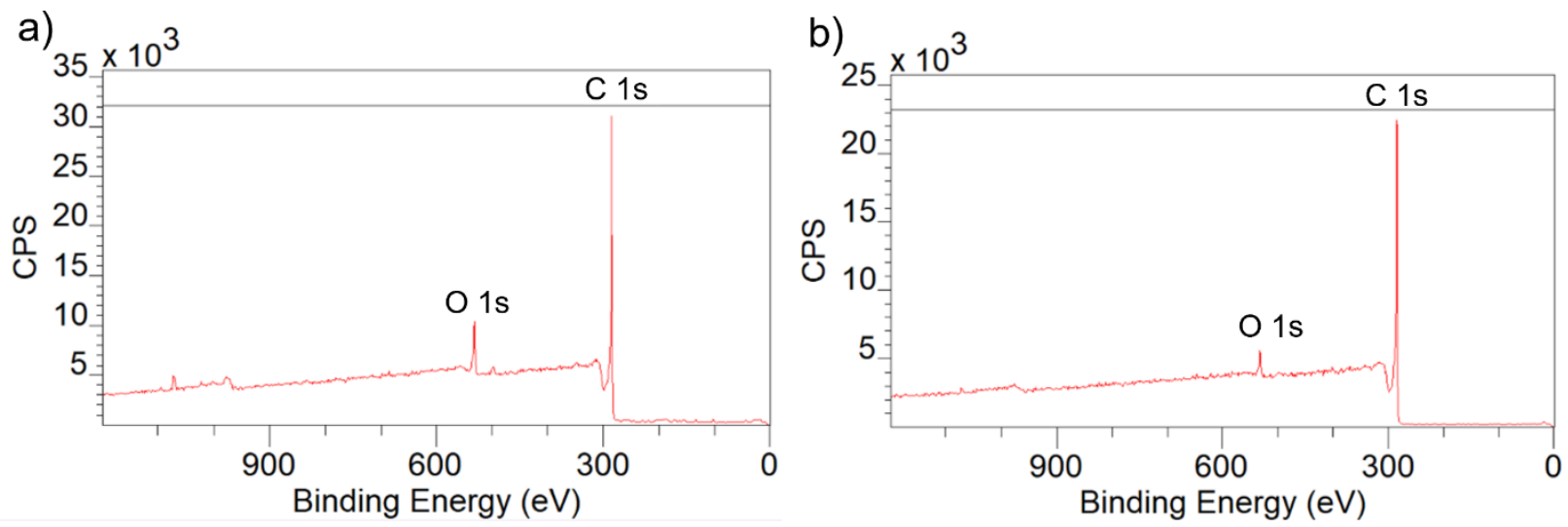

Figure S4: XPS elemental scans for a) ULPC-1 and b) ULPC-2 pyrolyzed at $900{ }^{\circ} \mathrm{C}$.

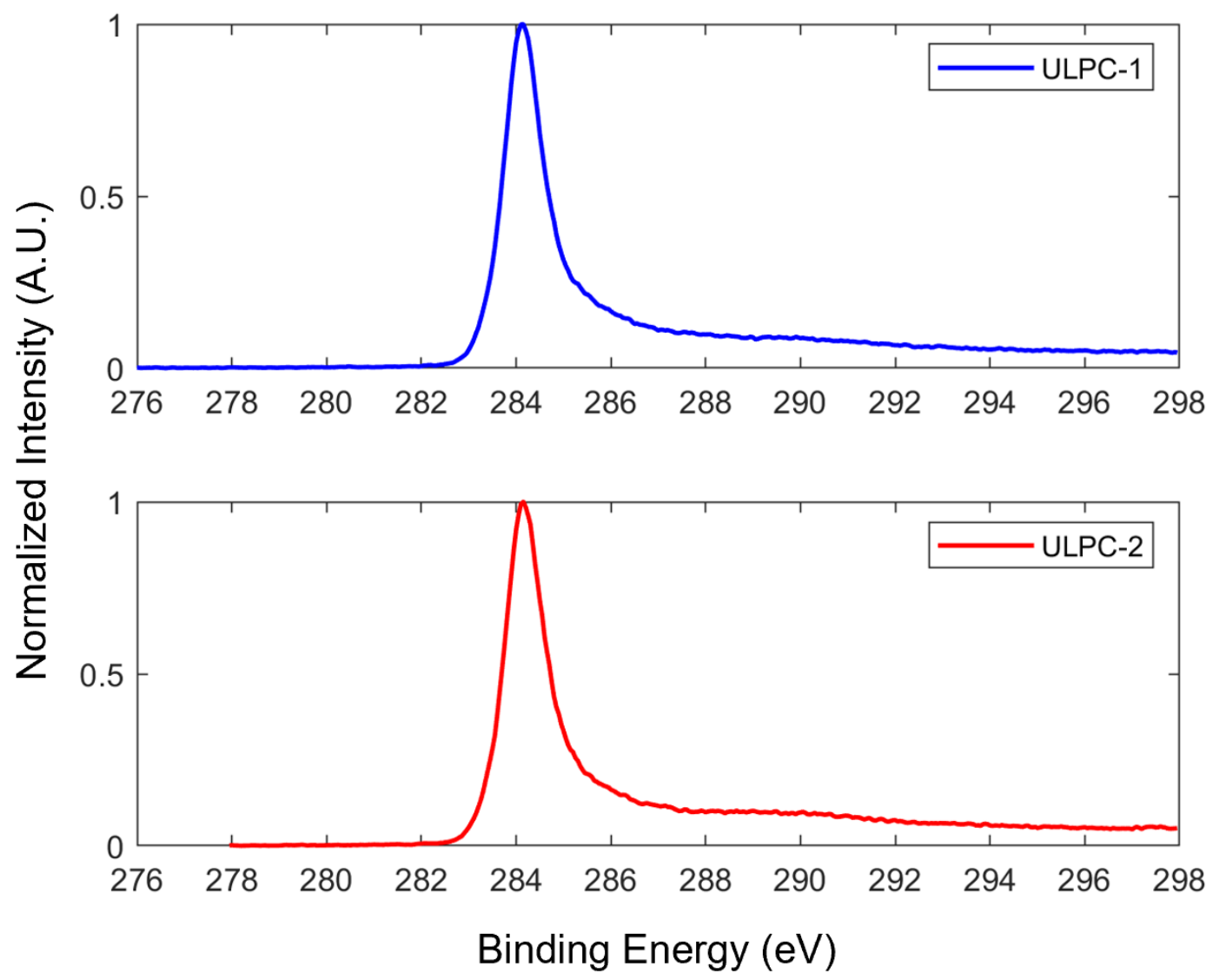

Figure S5: HR-XPS of carbon edge for ULPC-1 (blue) and ULPC-2 (red) pyrolyzed at $900{ }^{\circ} \mathrm{C}$ showing C1s peaks at $\sim 284 \mathrm{eV}$ with trailing tail towards higher binding energy, indicating $\mathrm{sp}^{2}$ hybridized carbon. 


\section{X-ray diffraction (XRD) data}

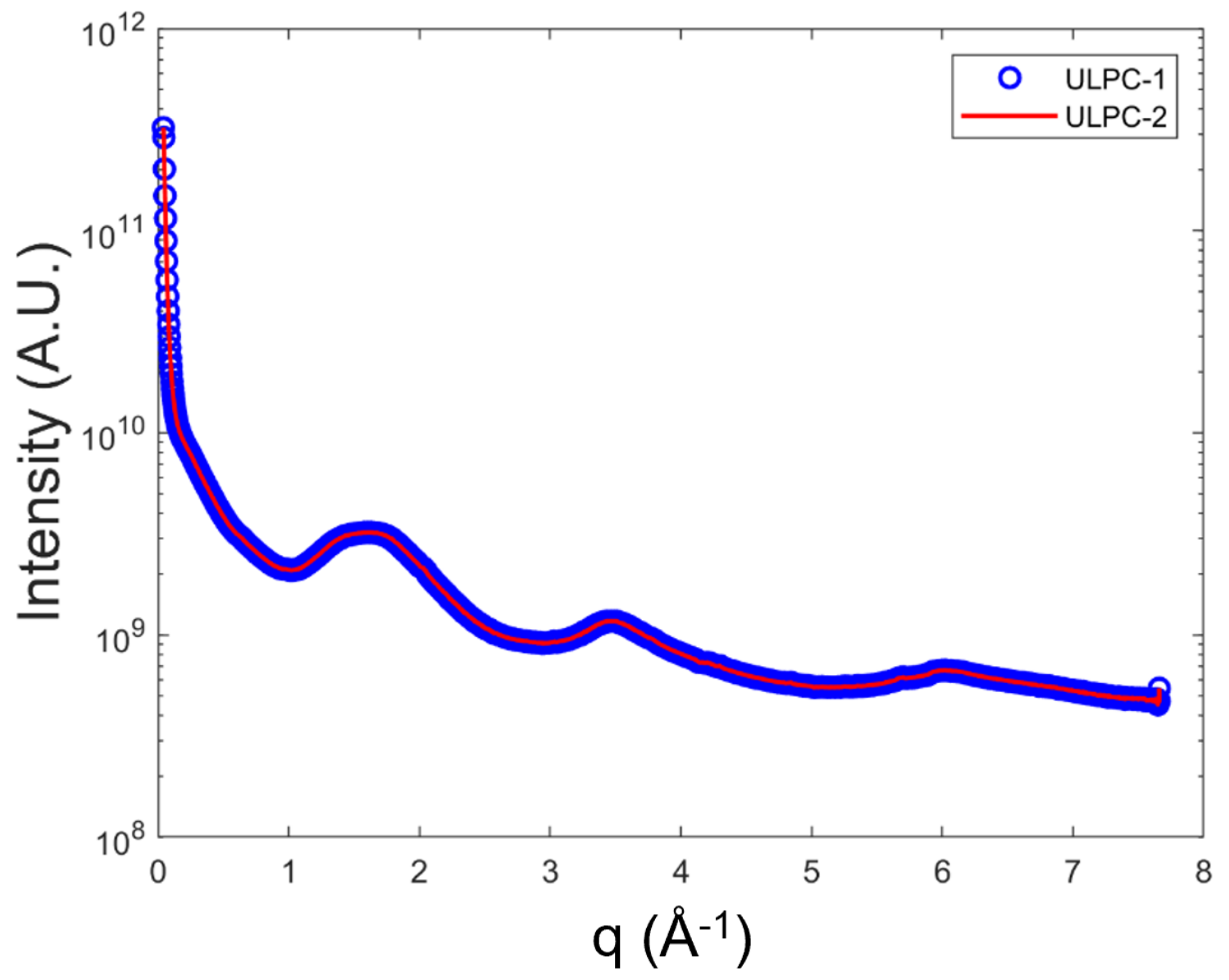

Figure S6: XRD results collected at the Soft Matter Interfaces beamline of the National Synchrotron Light Source, NSLS II, for ULPC-1 (blue) and ULPC-2 (red) pyrolyzed at $900{ }^{\circ} \mathrm{C}$. 